\title{
EXaMINE - Experimentation of a Monitoring and Control System for Managing Vulnerabilities of the European Infrastructure for Electrical Power Exchange
}

\author{
Antonio DIU \\ Red Electrica de España S.A. \\ Dirección General de Operación \\ Paral.lel 31, E-08004 Barcelona, Spain
}

\author{
Louis WEHENKEL \\ University of Liège \\ Dept. of Elec. Eng. and Comp. Science \\ Sart-Tilman B28, B-4000 Liège, Belgium
}

\begin{abstract}
This paper presents the overall goals and strategies of the EXaMINE project funded by the European Union (IST 2000 26116). The general objective of the project is to design, prototype and test novel monitoring systems supporting the control centers of the European power system in order to adapt and enhance their security control functions, thus allowing them to collectively meet the challenges of operating the European grid in a safe and reliable fashion, under increased interregional power transfers. The project considers security control in terms of its two complementary modes, namely preventive control and emergency control. Both aspects are tackled by using the latest developments in information technology in order to improve the quality of the information used for security monitoring and control. Field tests will be done with the cooperation of the System Operators that support the project.
\end{abstract}

The EXaMINE project has started in early 2001 and is scheduled to complete in August 2003. The partners of the project are the following:

REE - Transmission System Operator (Spain)

AIA - Consulting and Engineering (Spain)

CESI - Consulting and Engineering (Italy)

SEMA Group - Software and Systems Supplier (France)

SRI - Consulting and Research (UK)

University of Genoa - Research (Italy)

University of Liege - Research (Belgium)

GRTN - Transmission System Operator (Italy)

University of Milano - Research (Italy)

\section{European electrical context at the beginning of the 3rd millennium}

As in many other places around the World, in Europe the electric power sector is undergoing a series of very important changes which will probably have very strong impacts on the reliability and security of supply. While the European Electric Power System (EPS) was traditionally considered as one of the most reliable interconnected power systems, these changes are likely to largely increase its vulnerabilities in the next few years.
Among these vulnerability factors, the most well known one is due to the restructuring of the electrical sector towards a market-based environment, following the European Community directive of 1996, demanding un-bundling, open competition and third party access to the transmission systems. This is widely increasing the system volatility and puts more stress on the transmission grid due to increased cross-border energy trade. A system with unlimited capacity of interconnection between State, Regional or even Zonal Electric Systems, in a market based pricing scheme will lead to a unique price of electricity in Europe. When the physical limitation of interconnection capacity is considered, different prices will appear and the market regulations will demand interconnection capacity for the amount of energy needed to balance the prices of energy in both sides of the interconnection.

Competence in the electric market has already produced a significant reduction in the prices of electricity to the final consumers, but in mid term will create a strong demand of interconnection capacity to cope with the amount of interchanges across the full Interconnected System (Europe and North Africa mainly) among Producers and Consumers.

In correlation with this, generating companies as well as transmission system operators are forced to operate with lower safety margins, the first in order to remain competitive with respect to other suppliers, the second in order to allow this competition to take place all over Europe. Needless to say that this tendency, which is already quite advanced, will continue to change power flow patterns, increase uncertainties, and reduce security of supply if care is not taken.

The second important factor of vulnerability is due to the physical changes taking place in the system. In particular, the EPS, which is composed of a set of interconnected grids (each one separately controlled by a national operator), is now being extended to East European and Mediterranean countries (by the progressive construction of the so-called Mediterranean ring). These newcomers into the European system generally have weaker systems and lower security standards, and hence it is of strategic importance to control all possible disturbances so as to avoid their propagation.

Within this structural context, there is also a strong pres- 
sure in most European countries to accelerate the replacement of large coal fired and nuclear power plants by smaller generation plants either of the combined-cycle gas-turbine type or in the form of renewable energy sources (mainly large wind-farms). This tendency will create new common mode disturbance sources in the system, and also modify the energy flow patterns very strongly. At the same time, power system expansion is hardly possible and above all more and more difficult to justify from an economic point of view (notably due to the un-bundling of generation and transmission and because of the increased uncertainty about future power flow patterns).

Furthermore, a very important specificity of Europe is the way the interconnection is organized and regulated. Essentially, the European Union has no structure nor legal power to enforce a common way of organizing the whole business. On the top-level, the European Directives provide common objectives, general guidelines and principles. On the bottom level, the precise implementation of these guidelines is sub-contracted to each country (or even to each autonomous subregion, in some cases), where the rules and laws are created in order to comply with the European Directives and, above all, to fit into the local legal system, political organization and economic context. This approach intrinsically created a very diverse set of ways and steps of implementing the restructuring process; there are (at least) as many Regulators as European countries, and about as many different regulations as Regulators. Also, there is no federal body playing a role such as FERC does in the US, and the model adopted for the coordination of the different TSOs is essentially based on multi-lateral negotiation and cooperation.

Finally, there is a strong impediment towards information exchange among different actors of the European energy sector. Indeed, for several reasons, the European electric power industries have traditionally been very cautious in terms of confidentiality and security of technical information about their system. One of these reasons is the fact that in many European countries terrorism has been, and in several places still is, very active. Another reason, re-enforced by the restructuring, is obviously related to the commercial sensitivity of much of the information that should ideally be shared among TSOs.

Besides that, many efforts are being taken among ETSO members (European Transmission System Operators organization, see http://www.etso-net.org/) so as to establish a fluid and secure communications system among them.

In top of these technical arguments, the European Society will never accept a reduction of the quality of the supply. In consequence to deal with the reduction of security it is necessary to obtain an improvement of the tools used to detect and correct lacks of system reliability.

Thus, the challenges of maintaining the reliability and survivability of the European electric supply system are not to be underestimated. The EXaMINE project is part of the efforts presently taken at the European level to meet these challenges. We will try to show that this work is in this respect complementary to the work going on in organizations such as ETSO and UCTE and other European research projects.

We refer the reader to the paper by Dr. Singh [1] of the same panel, for a more in depth elaboration on ETSO and European RTO perspectives and current concerns. About the development of a platform for on-line dynamic security assessment, market simulation and operator training, we refer to [2] which describes another European Community funded research project.

\section{Overview of the EXaMINE project goals}

When a power system is confronted with a quickly changing environment such as the one observed in Europe, if nothing else is changed, its reliability and security will rapidly decrease. Among the different alternatives which one can investigate in order to maintain security, the one which is investigated in the EXaMINE project concerns the improvement of the quality and quantity of information upon which security control is based. This concerns both the information used by engineers in the context of operation planning and on-line operation, as well as the information used by automatic closed loop emergency control devices.

Notice that these two aspects are essentially complementary, in the sense that operation (and operation planning) aim at taking preventive decisions from a few days to a few minutes ahead of time, so as to cope with the planned or the most likely scenarios, while emergency control aims at handling all the remaining, unanticipated events in a $\mathrm{cu}$ rative and fast enough way to avoid catastrophic consequences. Notice that, ideally, emergency control would trigger if and only if it is necessary, i.e. if and only if a disturbance has taken place which can not be absorbed in a satisfactory way by the natural dynamics of the power system. Because success of emergency control is generally strongly dependent on speed, it is normally treated by automatic devices and without human intervention in real-time. Because of the restriction in time and also because of the limitation of available emergency control algorithms and devices, not all security problems can be solved by emergency control. Also, most emergency control systems, when they act, lead to rejecting part of the generation and/or load connected to the power system; thus, emergency control actions should be used as rarely as possible in order to maintain an appropriate level of reliability. One can therefore see preventive security control as a way to complement emergency control. In some sense, the preventive control actions are made in order to solve those problems which either could not be solved in a satisfactory way by emergency control, or which would lead to too frequent interruptions of service if handled by emergency control. However, preventive control introduces additional constraints on the power flows through the system which inevitably lead to increased costs for the end-users of the 
system (suppliers and consumers).

Thus, the optimal tradeoff between emergency control and preventive control, which depends both on economic pressure and available technology, is moving as time goes on and as the pressure towards reduced prices increases and as new technology becomes available. This general trend is pushing the compromise towards more emergency control and less preventive control (see [3] for a more in depth elaboration on these compromises and a discussion of the technological trends in this context). Nevertheless, improvements are presently required in both contexts and the EXaMINE project considers improvements both for emergency control and preventive control. We will proceed by separately discussing these approaches and solutions investigated in these two parts of the EXaMINE project.

\subsection{EXaMINE Preventive mode approach}

Power system operation at the European level is carried out by a huge multi-agent distributed control system. The individual control agents are the different national control centers, and, in an extremely simplified view, their control actions consist of (or translate into) accepting or rejecting transactions over their system. The precise mechanism used for implementing these control actions depends actually on the particular TSO, and is not relevant for our discussion. What is relevant here is the information used and the criteria implemented on top of this information for making the decisions (accept or reject).

Present practice is as follows (we simplify the picture of course). Each TSO maintains a physical model (in realtime, and also in the operation planning environment) of his own power system. These models are based on a combination of three types of informations:

- Off-line data and first-principles from physics: the elementary state equations of the different physical devices and the measured parameters (impedances, inertias, standard deviations of measurement errors...), and the external equivalents provided by the neighbor TSOs or by a central agency such as UCTE.

- Real-time measurements provided by the conventional Supervisory Control and Data Acquisition (SCADA) function every few seconds.

- The forecasted and planned quantities (weather, load, maintenance schedules...).

The basic tool for exploiting this information in the TSO environment is the load-flow software (and its extended versions, the state-estimator and the static security assessment package), which are used in order to determine whether or not forecasted or actual network state (load, generation and topology) in a given context should be accepted or rejected. The basic criterion for rejecting a network state is the capacity of the network state to meet the Security Criteria previously agreed by Regulators, Operators and Market Agents. These security criteria are normally based on the analysis of the actual or forecasted operating state and under all the contingencies with a certain level of probability to occur. This leads to the following decision rule: a state, or a particular transaction, is rejected by a particular TSO if it would lead, or if it would lead potentially under some contingency in the system of the TSO, to an unacceptable operating state (voltages or flows out of their limits) of the system controlled and supervised by this TSO.

At the European level, transaction management is thus handled in a distributed way, where each TSO essentially focuses on his own power system, and uses accurate and up-to-date information only about his own system. Clearly, any power system engineer would agree with the claim that it would be better to provide to all these agents a coherent and complete real-time model of the whole European system. Thus, for example for the Spanish System Operator's decision making, it would be better to take into account in his load-flow model the real-time information concerning (part of) France, Portugal, Morocco... Also, when this load-flow software computes a post-contingency state resulting from the tripping of a $400 \mathrm{kV}$ line in Spain, it would be better to monitor the voltages and power flows not only in the Spanish system, but also in France, Portugal, Morocco... The question is how this can be achieved in the most cost-effective way, while complying with the confidentiality and security requirements of data exchange among TSOs, and while avoiding the problems of data duplication and maintenance overhead.

\subsubsection{Distributed load-flow (DLF)}

Clearly, one solution to the problem would be to create a Mega-RTO at the European level, on top of the national TSOs. The approach developed in the EXaMINE project is however different, and fits better to the collaborative architecture of the European organization. The idea consists of using a distributed architecture in order to run a distributed load-flow engine decomposed in as many servers as there are TSOs. Suppose, for the sake of explanation, that there are only two TSOs in the system. Then EXaMINE proposes to install a distributed load-flow server in each TSO's control room. These servers are, on the one end, coupled with the local EMS system, from which they receive realtime and forecasted information about the local power system, and, on the other end, connected to the remote server, via a fast enough communication link, through which they receive computation results upon request, in the form of interconnection flows and boundary node voltages. The behavior of this system is fully symmetrical: each server is responsible for solving the part of the load-flow equations corresponding to his own subsystem and is a client of the other server which delivers relevant information about the other system. Also, when the operator of one TSO runs a load-flow, say to simulate the tripping of one of his lines, the detailed results concerning his own system 
will be displayed to him only. On the other hand, if this contingency creates violations in the neighbor system, synthetic alarms can be generated by the remote server and sent to both operators in order to let them know that their is an inter-TSO problem which should be analyzed more in detail and which solution needs probably cooperation between the two operators.

Obviously, this scheme can be extended to any number of TSOs, each one equipped with a sufficiently powerful EXaMINE server. This architecture would require a communication infrastructure with point to point links connecting only direct "electrical" neighbors, i.e. having the same interconnection topology then the actual power system. Also, at least in principle, the scheme can be used as well for state-estimation and contingency analysis, and both in operation planning and real-time operation modes.

The strength of this proposal comes from the fact that it is perfectly coherent with the actual collaborative nature of system wide operation in Europe, needs only a minimal amount of information sharing and hence is not very demanding in terms of communication infrastructures (actually, most of the communication links which would be needed in this scheme already exist in the form of point to point high-speed communication links among the different European TSOs).

On the other hand, it remains to be shown that from a mathematical, numerical and technical point of view this solution is indeed sound.

\subsubsection{EXaMINE DLF tasks}

Concerning the distributed load-flow part of EXaMINE the following tasks will be carried out:

- Develop and test the distributed load-flow software in the laboratory.

- Develop the interfaces between this software and at least two different EMS systems (among which the Spanish TSO will be one).

- Install the EXaMINE servers in these EMS environments and operate them in parallel using an existing secure communication link.

\subsection{EXaMINE Emergency control approach}

The emergency part of the EXaMINE project focuses on the use of synchronized phasor measurement units (PMUs) and ultra-fast communication systems in order to achieve the following objectives.

- Complement conventional SCADA with an ultra-fast realtime SCADA system, providing a global picture of the system state with sufficient temporal and spatial resolution so as to allow decision making in the context of fast phenomena such as loss of synchronism and fast cascading line tripping.

- Develop instability detection algorithms working in realtime speed.

- Develop closed-loop measurement based emergency control algorithms, fast enough to avoid loss of synchronism.

\subsubsection{Ultra-fast SCADA}

Part of the project aims at evaluating the performances of existing PMUs and secured communication protocols so as to determine the accuracy and temporal resolution possible for the ultra-fast SCADA. In this part, tests will be carried out, in a first stage with a real-time simulator, and possibly in a second stage by the installation of a reduced number of PMUs on the Italian EHV grid, connected with the GRTN control center SCADA system through appropriate communication links.

The other part of the work aims at determining the optimal placement of phasor measurement devices and at providing the required adaptations of state estimation techniques in order to yield observability of the fast dynamics of the system. Notice that the notion of observability has a different meaning here than in classical static state estimation. Since the system will be used for the monitoring of fast transients, observability means here observability of the relevant fast state variables. For example, in the context of transient angle stability emergency control, this means essentially observability of the mechanical rotor angles of a yet to be determined subset of generators, and with a temporal resolution and latency in the order of 50 to 100 milliseconds. In the context of voltage stability emergency control this observability is referring to quantities such as voltage magnitudes and excitation currents, with probably a less demanding temporal resolution, and, in the context of fast cascading line tripping, observability is related mainly to power flows or currents in various lines.

\subsubsection{Instability detection}

Within the EXaMINE project's restricted time-frame the focus will be on voltage collapse and loss of synchronism. The development of detection algorithms will be based both on small test systems and on a simulation model of the full Italian power system.

As concerns, loss of synchronism the detection will be based on the emergency mode version of the SIME method (Single Machine Equivalent). This method is normally using rotor angles and other quantities related to all the generators in the system in order to monitor loss of synchronism. The first part of the work is focusing on the use of non-linear modeling techniques (such as neural networks) in order to estimate these angles from real-time Voltage and Current phasor measurements taken at the EHV side of the step-up transformer of a generator. The second step will 
aim at selecting, for the Italian system, a reduced number of power plants to monitor and at using this information together with the E-SIME method [4] for the early detection of loss of synchronism. Then, the method will be tested on the basis of full scale time-domain simulations incorporating in their model not only the power system dynamics, but also the PMU devices, the non-linear estimators (one neural net module, specifically trained for each monitored generator), and also the communication links and black box models of the ultra-fast SCADA. These tests will allow us to determine the reliability and the speed of detection, and decide whether the technique can be used for actual emergency control in the Italian system.

\subsubsection{Closed loop emergency control}

Concerning voltage collapse, an appropriate strategy for emergency control is presently under investigation.

As concerns loss-of-synchronism two approaches will be developed and tested in parallel.

- E-SIME based generator tripping. The method is explained in detail in ref. [4]. It is essentially a by-product of the E-SIME based instability detection. Indeed, the latter determines the critical group of machines, i.e. those loosing synchronism with respect to the rest of the system. The control module will trip part of these machines in a sequential closed loop fashion. It estimates, on the basis of the real-time single machine equivalent powerangle curves the amount of excess energy and how much of it can be cancelled by tripping each generator. Then the minimal number of generators to trip is determined and a tripping signal is sent to the corresponding power plant(s). The detection module is then called back in order to further monitor the rotor angles after the fact in order to check whether stabilization is successful or not. If not, the control module is called again with the refreshed information in order to decide on a new (additional) control signal to send, and so on.

- Reinforcement learning based control. This approach is investigated in the EXaMINE project from a more academic point of view, as a long term full fledged solution for emergency control. Reinforcement learning methods provide an automatic way to learn how to control a system (by trial and error) in order to maximize a certain objective function. The method can be coupled either with a real system or with a simulation model of a system. This latter solution allows one to avoid destroying the real system at the early stages of learning, when the algorithm is still ignorant about the correct way to do control. It allows one also to set up appropriate simulation scenarios which are not normally observed on the real system but under which the control algorithm should work. Thus, in the context of electric power systems the learning stage is of course carried out on the basis of simulations. We refer the interested reader to [5] for a more in depth presentation of reinforcement learn- ing in the context of power system control. We merely note here that this is a very general method which is in principle able to handle the stochastic optimal control problem of any Markovian type of system, and to some extent also copes with reduced observability. Notice also that reinforcement learning can as well be used to provide approximate solutions in reasonable time to many stochastic large scale dynamic programming problems which exact solution is intractable.

From a practical point of view, we will connect a reinforcement learning module with the SICRE time-domain simulation engine developed by CESI for the Italian system, and test reinforcement learning in the context of electromechanical dynamics (damping and loss of synchronism) while investigating control actions complementary to those handled by E-SIME. In particular, we will consider the use of load-shedding and, in a more longer term view, the use of FACTS such as TCSC (Thyristor controlled series compensation) as possible control resources.

\subsection{EXaMINE project schedule}

The scheduled project duration is 30 months. The project started effectively in February 2001, and will be past midterm when this panel will meet.

At the time of writing this paper, the first two technical Work-packages have been successfully completed. They concern respectively the detailed needs expressions in both parts of the project and their technical specifications, including risk analysis.

The other two technical Work-packages concern respectively the software and prototype development, and the experiences analysis and evaluation. Concerning the first of these two, the work has already started in both parts of the project. In the context of the distributed load-flow application, software development has already significantly progressed, and it is expected to have a first prototype working by the time the panel will meet.

In the context of emergency control, PMU specifications from various suppliers have been analyzed and the final selection will also be done by the time the panel meets. Software development has also started, in particular in the coupling of the SIME software with the SICRE time-domain simulations. Also, some theoretical work has been carried out in the context of optimal placement of phasor measurement devices [6] as well as some first tests with the E-SIME method on the Italian system.

\section{Conclusion}

The work carried out in the EXaMINE project is essentially focusing on improving the information used for security control, so as to tackle the new difficulties introduced 
in this context, in general throughout the World, but also more specifically within the European interconnection.

In this goal, two complementary approaches are considered, referring, on the one hand, to the development of a mechanism and software framework to share information among TSOs in the preventive mode (the distributed load-flow approach), and the development of a hardware and software infrastructure to provide an ultra-fast SCADA which can support automatic decision making in the context of emergency control.

We believe that these efforts and approaches are complementary to the work going on in that context throughout the World, and that they may offer viable solutions to maintain the reliability of the European system at its past standards without conflicting with other objectives such as confidentiality, safety and economic competition.

\section{Acknowledgments}

The authors of this paper would like to express their grateful recognition to all the participants of the EXaMINE project (too numerous to cite explicitly here), as well as to their Companies and Institutions and to the European Commission which collectively are funding this project.

\section{REFERENCES}

[1] N. Singh, RTO: a cooperative model in the European context, IEEE Power Engineering Society Summer Meeting, Panel on Power system security in the new market environment, Chicago, 2002.

[2] S. Massucco, M. Pavella, OMASES: an approach to preventive DSA and control, IEEE Power Engineering Society Summer Meeting, Panel on Power system security in the new market environment, Chicago, 2002.

[3] L. Wehenkel, Emergency control and its strategies, 13th Power Systems Computation Conference (Plenary talk paper), Trondheim (Norway), 1999.

[4] Y. Zhang, L. Wehenkel, M Pavella, A method for realtime transient stability emergency control, Proceedings of IFAC Cigré Symposium on Control of Power Systems and Power Plants, Bejing (China), August 1997.

[5] D. Ernst, L. Wehenkel, FACTS devices controlled by means of reinforcement learning algorithms, to appear in the Proceedings of the 14th Power Systems Computation Conference, Sevilla (Spain), 2002.

[6] G.B. Denegri, M. Invernizzi, and F. Milano, A deterministic approach in positioning PMU for linear static state estimation, Technical report, University of Genova, Department of Electrical Engineering, August 2001.

\section{Biographies}

Antonio DIU was graduated Electrical Engineer by the Polytechnic University of Barcelona in 1971, and has finished the Business Administration School program of the University of Navarra IESE, in 1991. He is presently Manager of International Projects in the Operation Area of Red Electrica de España (Spanish transmission system operator). Mr Diu is also the Spanish representative in the CIGRE Study Committee 39 (Power System Operation) and is chairing the Working group 39.04 (Bulk Electric System Performance and Economic Assessment). He is the technical and administrative coordinator of the EXaMINE project.

Louis WEHENKEL graduated as an Electrical Engineer (Electronics) from the University of Liège, in 1986, received his PHD in 1990 and the "Agrégation de l'Enseignement Supérieur" in 1994. He is presently Professor in the Department of Electrical Engineering and Computer Science of the University of Liège, where he coordinates a research group composed of about 20 researchers. Dr. Wehenkel's research interests lie in the fields of stochastic methods, power system security control and planning, and the application of the former methods to the latter problems. He is convener of the CIGRE Task Force 38.02.21 on Security Assessment. 